\title{
Numerical study on deflagration and combustion effect of aircraft impact
}

\author{
Junwei Zhang ${ }^{1, a}$, Wenxiang $\mathrm{Cai}^{2, \mathrm{~b}}$,Ling $\mathrm{Yu}^{3, \mathrm{c}}$ \\ ${ }^{1}$ Nanjing University of Science and Technology, Jiangsu 210094, China; \\ ${ }^{2}$ Nanjing University of Science and Technology, Jiangsu 210094, China. \\ ${ }^{3}$ Nanjing University of Science and Technology, Jiangsu 210094, China. \\ a774058678@qq.com, bcaiwx_2005@njust.edu.cn, 'yuling61@mail.njust.edu.cn
}

Keywords: Deflagration, Numerical simulation, CFX, pressure.

\begin{abstract}
In the survey stage of nuclear power plant site, it is necessary to consider the impact on nuclear power station brought by the aircraft. The most serious consequence is the fuel leak. The fuel combustion process is divided into two stages of deflagration and pool fire. This paper is mainly focus on the simulation of deflagration and pool fire effect after the impact caused by aircraft impacting the architectural workshop, based on general computational fluid dynamics software CFX, using the method of numerical to analyze the duration of the deflagration and pool fire stage, transient temperature, pressure, changes in fuel concentration, and the effects of different impact height on deflagration and pool fire combustion process. The results show that, during deflagration and pool fire combustion period, the influence of temperature on the structural strength of buildings is low, so is the influence of pressure.
\end{abstract}

\section{Introduction}

In the stage of surveying the nuclear power plant site, it is necessary to consider the potential danger posed by the plane hitting the nuclear power plant. The most serious consequence among them is the escaping of the fuel carried by the aircraft. According to the distribution of fuel, status, etc., it may cause combustion of part or all of the fuel. Its combustion process can generally be divided into two stages, which are deflagration and fire pool. In the stage of deflagration, flame at high speed will promote the formation of a pressure wave, which causing a great pressure shock wave to the building, immediate high temperature also generates to a rather great thermal load in radiation and convection form. In pool fire stage, stable, long-time burning transfer huge amount of thermal energy to the reactor shielding plant, by convection, heat radiation in the form causing significant reduction the structural strength of the related parts. Whether deflagration or pool fire both require systematic research about the burning strength, range of action, duration and regular distribution, and analysis of effect from the stress wave, the radiation, the influence of heat convection to buildings.

\section{Object of the Study}

Physical Model. This paper's simulated reactor is showed below. The height of the reactor is approximate $91.5 \mathrm{~m}$, the part above the ground is about $75.6 \mathrm{~m}$, and the diameter is about $48 \mathrm{~m}$.

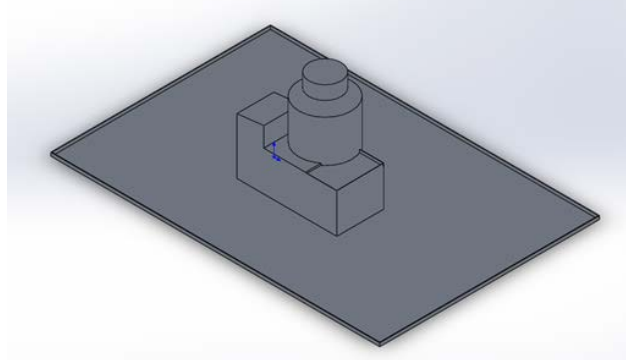

Fig. 1 physical model

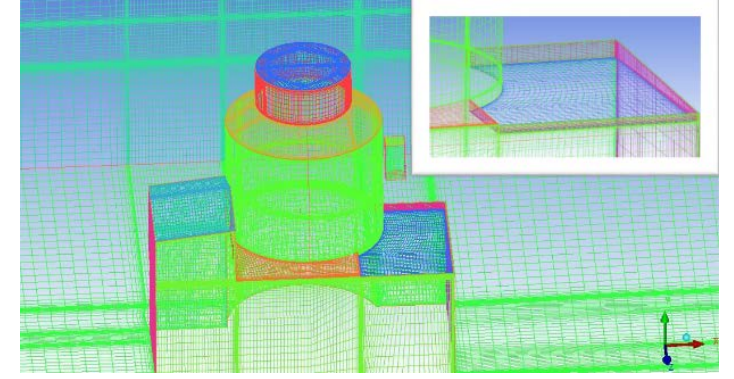

Fig. 2 architectural and local mesh of roof's oil bath 
Research Contents. This paper quasi-studies on the effect of pressure and heat load strength to reactor shielding plant from different proportion of fuel deflagration. Here, it is assumed the ratio of exploding oil to total remaining oil are 1/20,1/10,1/5 and 2/5 respectively, After assuming the weight of the deflagration fuel, based on factors such as fuel properties, environmental conditions and so on, it is feasible to analyze the range of initial combustible fuel-air mixture, gas-oil ratio and other parameters by calculating the fireball deflagration process, on the one hand, it is feasible to calculate the duration time of fireball explosion; on the other hand, it's feasible to get the impact of the process of the reactor by the pressure and temperature shock through calculation. In the process of the aircraft hitting the reactor, the position of the point of impact plays greatly significant role on the size of the fireball explosion and distribution of the fuel pool fire. This paper quasi-analyzes on the deflagration effects of an impact on the 1/3,1/2 and 3/4 the height of the reactor, considering the impact of semi-confined space of deflagration effects.

In this paper, numerical simulation of pool fire effect is based on the assumption of the even distribution of the fuel, using in pool fire, within the walls of the station, According to the fuel weight needed in fuel pool fire effect with the combination of physical properties of the fuel factors, environmental conditions and other calculated physical properties such as fuel evaporation rate parameters, using numerical methods to obtain pool fire burning time and the pool of fire burning intensity. On the one hand, we can calculate the pool of fire burning duration, transient pressure and temperature distribution, on the other hand it is feasible to calculate plant reactor shielding wall surface temperature and pressure in the associated process, considering the cumulative effects of the heat load and the effect of pool fire combustion of reactor shielding plant.

\section{Model and method}

Turbulence Model. In this paper, the calculation of flow field all adopt SST k- $\omega$ model which is based on the shear stress transport, and is proposed by Menter. The model owns following characteristics: possessing k- $\omega$ turbulence model near-wall features and advantages of k- $\varepsilon$ turbulence model in the free stream calculation; considering the transmission of turbulent shear stress which can accurately predict that the amount of separation at the start of the fluid flow conditions and in the negative pressure gradient, especially for simulative applications which require high precision boundary layer.

Boundary Conditions. Model grid is shown in Fig. 2. It is assumed that the natural wind blows from right side of the reactor at a speed of $1 \mathrm{~m} / \mathrm{s}$ and its outbuildings.

\section{Calculation Results Analysis}

Assuming massive pool fire burning phenomenon occur on the building, with a fence, which located at the right side of the reactor, the result simulated by the rate of evaporation of fuel therein, is input as the import conditions Deflagration occurred in the three-dimensional region $2 \mathrm{~m} \times 2 \mathrm{~m} \times 6 \mathrm{~m}$ square which is on the right of the reactor, inside of it contains a mixture with equivalence ratio of fuel and air.

Reactor main building is set as the center to establish a triangular rectangular coordinate, and three public monitoring points are set up, including public monitoring point 1 is located at $(28 \mathrm{~m}, 60 \mathrm{~m}, 0 \mathrm{~m})$; public monitoring point 2 located at $(28 \mathrm{~m}, 34 \mathrm{~m}, 0 \mathrm{~m})$, namely the roof surface of oil tank; public monitoring points 3 located at $(60 \mathrm{~m}, 1 \mathrm{~m}, 0 \mathrm{~m})$, it is the ground.

Numerical Analysis and Simulation of Deflagration and Pool Fire Combustion. Fig. 3 is the pressure change curves of deflagration and pool fire burning at the impact height of 1 , According to the graph, the time of impact of deflagration is very short, after a brief deflagration, pressure of all the monitor point and environment is quite basic. It can be explained that during the pool fire burning period, as the diffusion combustion process in an open space, the fuel in the oil pool on the roof is heated, evaporation and have chemical reaction with oxygen in the ambient inhalation of combustion, 
cannot form an effective boost or drop pressure process, resulting in roughly equal pressure with the surrounding environment. Therefore, the pressure load on the fire burning reactor wall in the pool fire stages is extremely small, even negligible. The pressure on the center of the oil and gas deflagration district (public monitoring point 1 ) is in the combustion of deflagration, showed in Fig. 4, in $2 \mathrm{~mm}$ to reach the peak pressure 7.5bar, at a breakneck speed and then led to over-expanded phenomenon, making its inner pressure maintaining a negative pressure for a certain time. After $0.1 \mathrm{~s}$ the negative pressure ambient pressure is roughly equal with the environmental pressure. High pressure wave reach the roof sump at 30ms, the oil pressure of the rooftop pool increased to 2.3bar, the latter part of the same phenomenon due to excessive expansion (negative pressure) occurs. It should be noted that, in the area of oil and gas deflagration position, the negative pressure caused by the over-expanded caused lead the fresh air around into the low pressure region so quickly, while the range near the oil tank roof is restricted, the supplement of the fresh air is not as quick as it in oil deflagration region, the maximum negative pressure in the low pressure region is slightly higher than that in the deflagration center region.

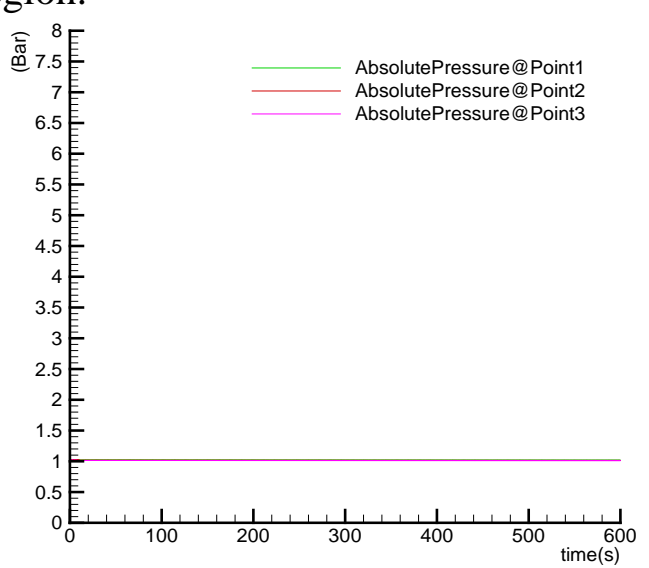

Fig. 3 pressure changes over time

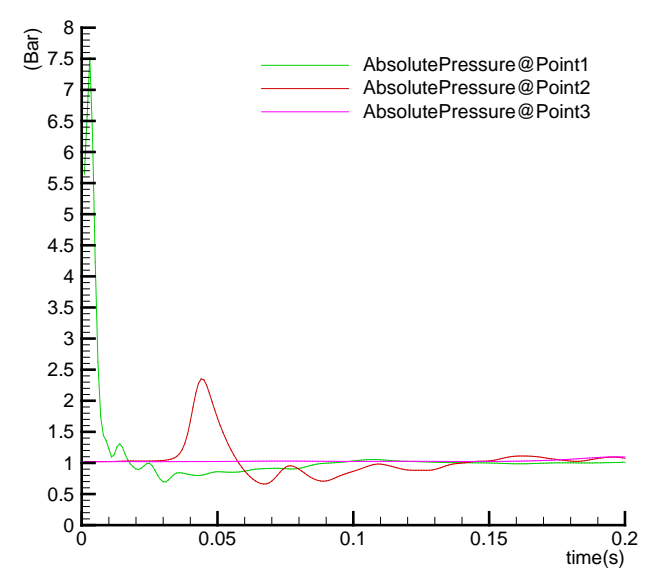

Fig. 4 pressure changes over $0.2 \mathrm{~s}$

Fig. 5 and Fig. 6 are the law of the temperature's change over time respectively in the calculation period and in the deflagration stage. The figure shows that the temperature in the deflagration region during the deflagration period basically reached its high temperature region (> 3000K) within 20ms, remaining for about $0.14 \mathrm{~s}$, and then quickly lowered to the fairly level close to ambient temperature. In the explosive period, the combustion time is extremely short, and the temperature of the public monitoring points within 2 and 3 almost remains unchanged. We can know from the variation curve of the pressure (Fig. 6) and temperature (Fig. 4) during the deflagration that the transfer rate of the heat of the high temperature gas is much lower than the spread of the pressure. This is because the fluid pressure wave spreads with the sound speed and the temperature mainly spread with convection and heat conduction, and the convection of the fluid always need some time from static to high-speed. It leads to an existence of temperature peak in the late deflagration. This is mainly because that the temperature of the gas is raised by the burning of the unburned fuel.

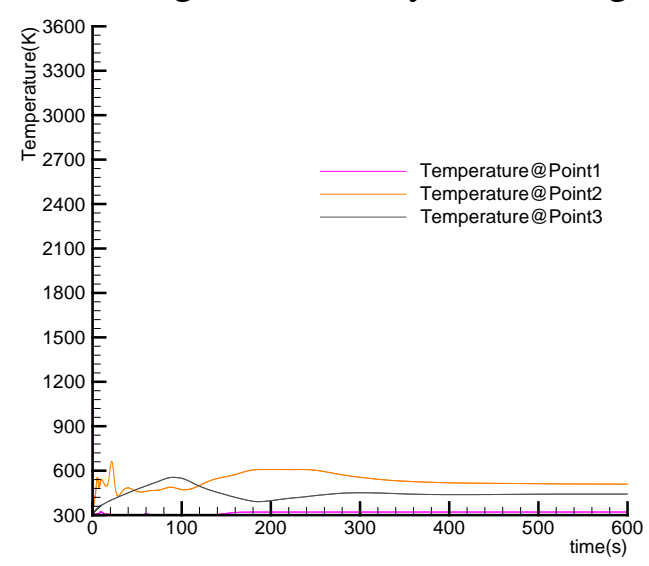

Fig. 5 Temperature changes over time

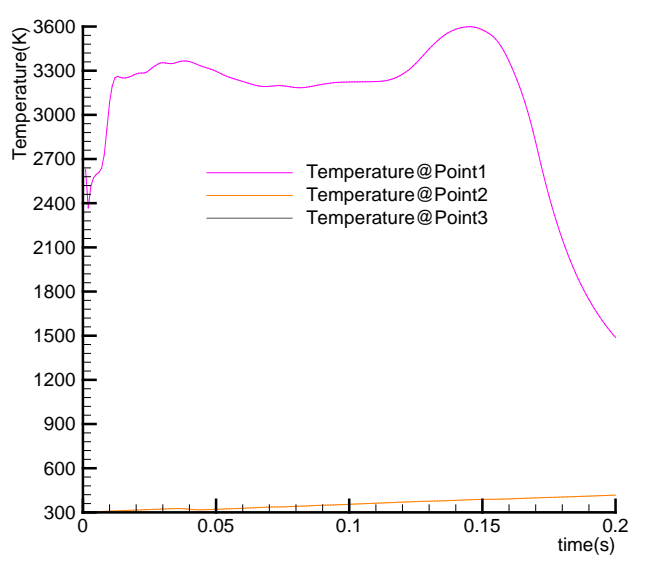

Fig. 6 Temperature changes over $0.2 \mathrm{~s}$ 
Effect of the impact height on deflagration and pool fire combustion process. In order to analyze the effect of different impact height on deflagration and pool fire combustion process, the oil and gas deflagration zone are arranged in three different locations. Impact height 1 (case 1) is the same as the impact height mentioned before. The upper edge of the impact point is $9 \mathrm{~m}$ lower than that of the equivalent diameter of the reactor. Impact height 2(case 2) is $8 \mathrm{~m}$ lower than impact height 1 and Impact height 3(case 3) is $8 \mathrm{~m}$ higher than impact height 1.

Table 1 is the peak value of temperature and pressure and the location of the monitoring points under different impact height. In deflagration combustion zone center, the air needed for deflagration combustion is mainly provided by the region itself, therefore, the deflagration is less affected by the surrounding environment. The temperature at point 1 in case 2 is relatively high. This is mainly because that in case 2, the gas in deflagration zone has certain diffusion under the pressure. The unburned out fuel rise and combust under the pressure, and heat transfer convection lead to that the temperature which is higher than that of center area of deflagration. For case 3, the fuel moves upward, but away from oil and gas deflagration zone, the effect of unburned fuel further combustion is relatively low, which makes the peak temperature to be lower than that in case 1 and much lower than that in case 2.

The propagation of deflagration pressure is mainly affected by the spatial range and the oxygen supplement. In the condition of 1 , the surrounding space is relatively open and can be replenished with oxygen relatively in time, so as to make the highest gas pressure. And in condition 2, the range is small, the deflagration pressure wave spread to some small, but fresh air replenishment rate is much lower, so the peak value of the gas pressure decreases. And for case 3, the energy produced by deflagration transfers in the most open space. Oxygen supplement is faster but its impact is relatively small, so that the inner pressure is lower. This shows that for the pressure wave produced by the deflagration combustion, influences of confined space are greater than that of the oxygen diffusion. Through above analysis, it is known that the peak value of the pressure wave generated by the position of the middle of the reactor is maximum when the aircraft hit the reactor.

Table 1 peak value of temperature and pressure at different impact height and the location of its monitoring points

\begin{tabular}{cccc}
\hline case & Case 1 & Case 2 & Case 2 \\
\hline peak value of temperature (K) & 3600(point1) & $3800($ point1) & $3300($ point11) \\
peak value of pressure (bar) & $7.5($ pint1) & $6.9($ poiny11) & $6.4($ poiny11) \\
\hline
\end{tabular}

\section{Summary}

The impact of gas temperature on the strength of building structure.

a) The peak pressure caused by the deflagration combustion is about 7atm. Pressure is rapidly reduced in the form of pressure waves within 3ms and negative pressure is created in the oil and gas deflagration zone after the deflagration pressure peak.

b) The temperature is kept at high temperature within $0.15 \mathrm{~s}$ in the deflagration zone, but the temperature decreases at a rather fast rate after the deflagration. In General, temperature transfer is limited by convection diffusion, which can maintain a longer time compared to the pressure. The area affected by high temperature which is created by the deflagration is relatively small and the time is relatively short. Considering the own thermal resistance of the building, the heat transfer takes a long time. So it can not affect the structural strength of the building in a short time. The pool fire combustion after the deflagration is diffusion combustion and the combustion is not sufficient. The combustion time is long but the gas temperature is relatively low. Therefore, the influence of temperature on the structural strength of buildings is relatively low in the process of pool fire and deflagration.

\section{The impact of gas pressure on the strength of building structure.}

a) The peak value of deflagration pressure wave reduced quickly in the process of transfer. The time of effect of the peak pressure on the surface of the building is very shot and the range of the 
impacted region is roughly the same size as the deflagration zone. The pressure has decay to slightly higher than the pressure on the environment when the influence area of the deflagration pressure wave spread to most of the surface of the building.

b) The scope of combustion is related with the surrounding environment at the pool fire stage. There is a little change of pressure which is caused by the low amplitude pressure pulsation created by the turbulent combustion in the pool combustion area and the impact on the building structure strength is negligible. The peak pressure created by the deflagration is relatively large and the force on the surface of the building is very short at the oil and gas deflagration zone, while the time of pool fire combustion is relatively long but the pressure change is relatively small. Therefore, the forces acting on the surface of the building are small in the whole calculation period and the influence of deflagration and pool fire combustion on the building structure strength is relatively small.

\section{Acknowledgments}

The authors gratefully acknowledge the sponsorship of Project 51306092 supported by National Natural Science Foundation of China, "Mechanism research of pulse combustion and its pollution emission".

\section{References}

[1] NUREG-1824, EPRI 1011999 Verification and Validation of Selected Fire Models for Nuclear Power Plant Applications [R]. U.S. Nuclear Regulatory Commission, Electric Power Research Institute, 2007.

[2] Liu Xiaoshuang, Xiao Jun, Bi Kun, Yang Guanghua, He Le. Study on application of numerical simulation in the fire protection design of nuclear power plants [J]. Journal of Safety Science and Technology, 2013.

[3] Huang Xianjia, Tu ran, Yi Jianxin, et al. The main scientific challenges in fire safety research of nuclear power plants [J]. Journal of University of Science and Technology of China, 2013, 43(11):959-966.

[4] Miao Yudong, Xu Shuo. Numerical simulation of natural ventilation fire in industrial plant [J]. Energy Conservation, 2007.

[5] Rogers K A, Fire in the hole: A review of national spent nuclear fuel disposal poliy [J]. Progress in Nuclear Energy, (2009),51[2]:281-289.

[6] Sun Zhiyou, Study on combustion characteristics of aviation kerosene pool fire [D]. University of Science and Technology of China, HeFei, (2008).

[7] Zhang Shuo, CFD simulation on burings rates and radiation distribution of open pool fires[J], Fire Science and Technology.(2011).

[8] IEAE, Experience Gained from Fires in Nuclear power plants: Lessons Learened [R]. USA International Atomic Energy Agency.(2004). 